\title{
MBC2019: Marine Biotechnology Conference 2019
}

\author{
Haruko Takeyama ${ }^{1,2,3} \cdot$ Hiroshi Saito ${ }^{4}$ Y Yohei Nishikawa ${ }^{2,3}$ \\ Published online: 26 November 2020 \\ (C) Springer Science+Business Media, LLC, part of Springer Nature 2020
}

As the developments in molecular biology, the field of marine biotechnology is currently undergoing a dramatic period of change. In particular, the development of big data acquisition and analysis technologies including the next-generation sequencers has led to the acquisition of various knowledge and the creation of new research topics. At the same time, we are faced with a variety of problems including marine microplastics that have not been previously identified. Thus, we are now expected to contribute to play an important role not only in advancing and disseminating research but also in industries and policies under the industry-governmentacademia collaboration. To provide a cross-sector forum for researchers from around the world to meet and discuss these various research topics from a professional point of view, the Marine Biotechnology Conference 2019 (MBC 2019) was held at Shizuoka City, Japan (Marinart Shizuoka City Shimizu Cultural hall, Shimizu Terrsa), from September 9 (Monday) to September 13 (Friday), 2019, by the Japanese Society for Marine Biotechnology (JSMB) which has more than 30 years of history.

Haruko Takeyama

haruko-takeyama@waseda.jp

Hiroshi Saito

cansai@scc.u-tokai.ac.jp

1 Department of Life Science and Medical Bioscience, Waseda University, 2-2 Wakamatsu-cho, Shinjuku-ku, Tokyo 162-8480, Japan

2 Computational Bio Big-Data Open Innovation Laboratory, AIST-Waseda University, 3-4-1 Okubo, Shinjuku-ku, Tokyo 169-0072, Japan

3 Research Organization for Nano \& Life Innovation, Waseda University, 513 Wasedatsurumaki-cho, Shinjuku-ku, Tokyo 162-0041, Japan

4 School of Marine Science \& Technology, Department of Fisheries, Tokai University, 3-20-1, Orido, Shimizu-ku, shizuoka-shi, Shizuoka 424-8610, Japan
The MBC2019 was a joint conference of the 12th International Marine Biotechnology Conference (IMBC) and the 12th Asia Pacific Marine Biotechnology Conference (APMBC). In 2003, the joint conference of both international conferences was also held in Japan (Makuhari, Chiba). At that time, more than 700 participants from 32 countries attended $\mathrm{MBC} 2003$, and fruitful and productive discussion in a wide range of research fields was conducted. After an interval of 16 years, the joint conference MBC was held in Japan again.

As a whole, 545 people attended the conference throughout the 5 days, with about $40 \%$ of the participants coming from overseas including 27 countries and regions (Fig. 1). MBC2019 consisted of eight plenary lectures, 37 invited speakers, more than 100 oral speakers, and more than 260 poster presentations including 15 sessions ((1) Disease and Immunology in Aquaculture, (2) Blue Innovation, Science to Policy, (3) Algal Biotechnology, (4) Natural Products from Marine Bioresources, (5) Reproductive and Developmental Technology in Aquaculture, (6) New Analysis for Microbial Community: Metagenomics to Single Cell Analysis, (7) Marine Biotechnology for Environmental Conservation, (8) Marine Functional Foods, (9) Innovative Approach to Sustainable Aquaculture and Fisheries I, (10) Marine Microbiology and Biotechnology, (11) Marine Genomics and Bioinformatics, (12) Biogenic Materials and Mineralization, (13) Energy and Material Recycle by Marine Biotechnology, (14) Innovative Approach to Sustainable Aquaculture and Fisheries II, and (15) Marine Symbiosis \& Ecology). We had prepared not only plenary lectures, invited lectures, general lectures, and poster presentations but also a variety of presentation formats including short oral presentations for student poster presenters and a night-time plenary in which speakers and audience members could talk over drinks and snacks. The elaborate program allowed us to close the MBC 2019 with great success.

Of a wide variety of topics MBC2019 dealt with, this Special Issue includes some examples of the species, research topics, and technologies in 10 papers. A study related to 


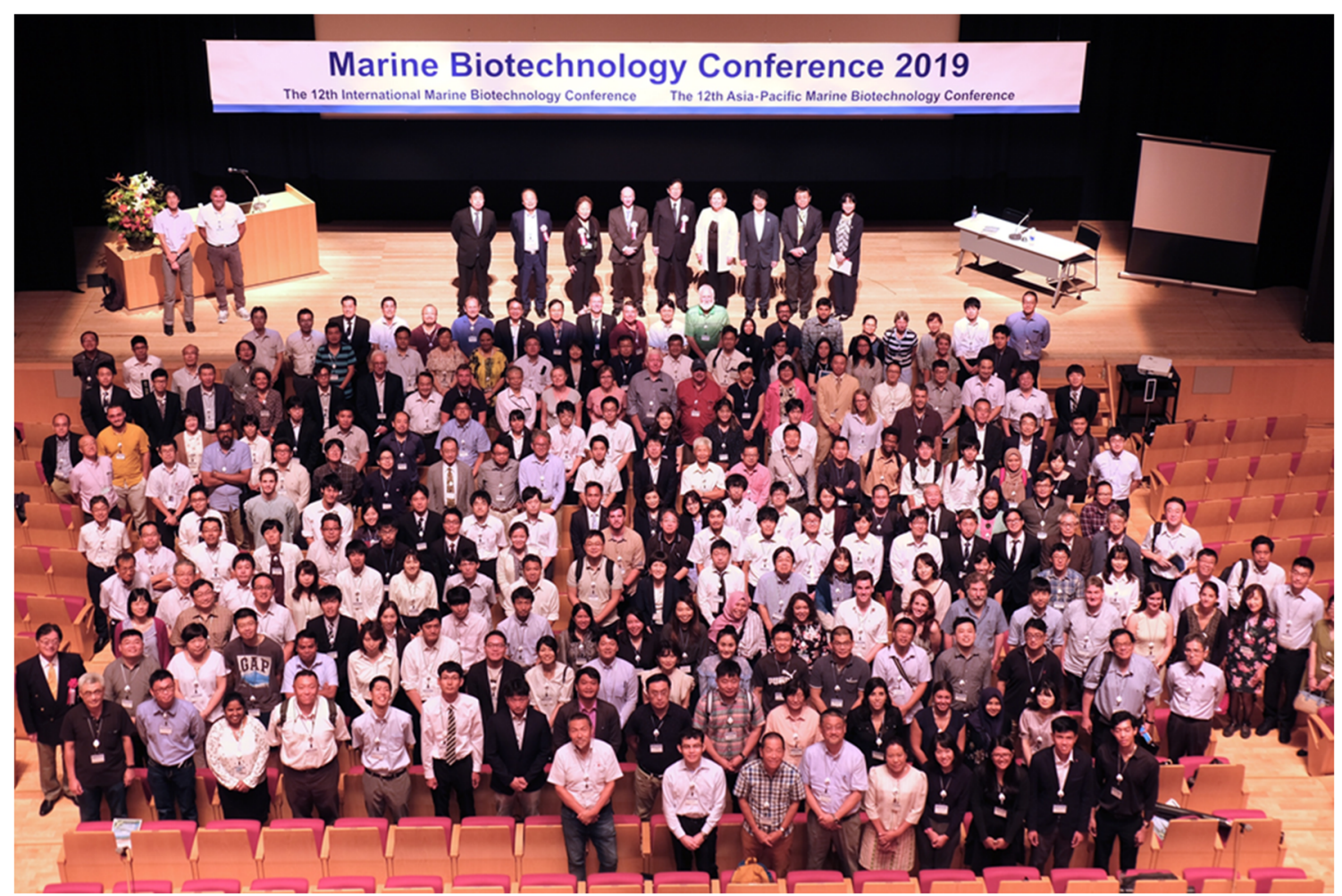

Fig. 1 Photograph at the opening ceremony of MBC2019. Executive members on the stage and participants at the floor to the ceremony held on the first day of MBC2019

Global Environment and Microbiology was presented by Kerfahi et al., which compared the biofilm bacterial communities on intertidal cobbles/boulders and bedrock along a seawater $\mathrm{CO}_{2}$. A study related to biomineralization was presented by Nishi et al., which clarified the domain responsible for the promotion of silica formation. Genomic and transcriptomic analysis was presented by Takagi et al., Yoshida et al., and Huang et al. Takagi et al. conducted transcriptome analysis in primary polyp of coral Acropora digitifera. Yoshida et al. conducted a genomic and transcriptomic analysis of a sparkling enope squid to clarify the molecular mechanism underlying its scintillation. Huang et al. presented the first transcriptomic analysis to assist freshwater ornamental shrimp germplasm enhancement and its extensive applications. In the field of fisheries, Nguyen et al. conducted the gut microbiota profiling of Atlantic salmon to assess the correlation of the gut microbiota with the flesh color. Kashitani et al. investigated taxonomical distribution patterns of Tetrodotoxin (TTX) by molecular phylogenetic analysis and high-performance liquid chromatography-tandem mass spectrometry (LC-MS/MS). Okabe et al. investigated the genetic structure of Landlocked Salmon populations in the Japanese river by sequencing the mitochondrial control region. In the field of Algal biotechnology, Nuryadi et al. characterized the filamentous cyanobacteria from Okinawan coasts as potential sources of bioactive compounds. Saito et al. evaluated the areal biomass productivities of marine chlorophyte, Nannochloropsis.

While this Special Issue publishes only some examples of the research papers presented at MBC2019, we are confident that MBC 2019 was a great success. "Next-generation Marine Biotechnology" was the main theme of the conference. We believe that we could provide a place for researchers to interact actively and promote global research exchange and industry-government-academia collaboration. We also hope that the conference has promoted the development of young researchers. We would like to appreciate the executive committee in IMBA, members at large, board members in APSMB, and 17 members of the local committee for their great efforts in planning and organizing the conference. We would also like to express our sincere gratitude to the people of Shizuoka Prefecture, Shizuoka City, and other local organizations and companies for their support in the preparation for and during the conference.

We would like to thank the sponsors, organizers, invited speakers, and all the participants for the great success of MBC2019. We would also like to express our gratitude to Marine Biotechnology for publishing this Special Issue.

Publisher's note Springer Nature remains neutral with regard to jurisdictional claims in published maps and institutional affiliations. 